\title{
Clinical challenges in the management of endocrine side effects of immuno-oncological therapies
}

\author{
Peter Wolf · Thomas Scherer
}

Received: 30 August 2021 / Accepted: 18 October 2021 / Published online: 24 November 2021

(C) The Author(s) 2021

\begin{abstract}
Summary Given the growing use of immune checkpoint inhibitor (ICI) therapy in oncology, the prevalence of endocrine side effects is rapidly increasing. As clinicians are nowadays frequently confronted with these side effects in routine clinical care, awareness, better knowledge of endocrine irAEs and their clinical presentation and diagnosis is crucial for an adequate management. In this short-review we give a compact overview of the recent recommendations for the management of endocrine irAE related to ICIs and highlight difficulties and uncertainties in current clinical practice.
\end{abstract}

Keywords Immune related adverse effects . Hypophysitis · Thyroiditis · Anti-CTLA4 · Anti-PD1

\section{Background}

The inhibition of immune checkpoints by using monoclonal antibodies against cytotoxic-T-lymphocyteassociated antigen 4 (CTLA-4) or programmed cell death 1 (PD-1) has revolutionized anti-cancer therapy in recent years and emerged as a cornerstone in the treatment of a constantly growing number of solid cancer types. CTLA-4 and PD-1 receptors both regulate cytotoxic T-cell activation and anti-tumor immune responses by distinct mechanisms. Antibodies blocking these immune regulatory pathways (immune checkpoint inhibitors [ICIs]) allow to restore anti-tumor immunity and thereby promote tumor cell death [1]. However, the immune system activation triggered by ICIs is not solely restricted to the tumor, which

\section{P. Wolf $(\varangle) \cdot$ T. Scherer}

Department of Internal Medicine III, Division of Endocrinology and Metabolism, Medical University of Vienna, Währinger Gürtel 18-20, 1090 Vienna, Austria peter.wolf@meduniwien.ac.at explains the autoimmune side effects often provoked by these medications. These immune-related adverse events (irAE) are markedly different from side effects seen in classical chemotherapy $[2,3]$. Endocrine irAEs associated with ICI therapy have a prevalence ranging from about 3 to 20\%, varying also depending on the type of ICI and/or if given in combination $[4,5]$. The risk is highest when anti-CTLA4 and anti-PD1 therapy is combined and appears less frequent in anti-PD1 ligand monotherapy [1].

Given the growing use of ICI therapy in oncology, clinicians are increasingly confronted with these side effects in routine clinical care. Awareness, better knowledge of endocrine irAEs and their clinical presentation and diagnosis is crucial for adequate management. In this short review we give a compact overview of the recent recommendations for the management of endocrine irAE related to ICIs and highlight difficulties and uncertainties in current clinical practice.

\section{Thyroiditis}

ICI-related thyroid dysfunction is the most common endocrinopathy caused by an irAE. It is more frequent in patients receiving anti-PD-1 directed treatment [6], probably due to the high expression of PD-1 receptors in thyroid cells [7]. In most cases, it results from a silent, painless immune-mediated acute inflammation, which leads to destruction of the thyroid gland, but Grave's disease and endocrine orbitopathy have also been described in rare cases. Inflammatory destruction releases preformed and stored thyroid hormones from thyroid follicles inducing transitory hyperthyroidism, which is often followed by chronic hypothyroidism [8]. Clinical signs and symptoms are often mild. However, some patients might develop thyrotoxicosis. The diagnosis is mainly based on the pa- 
tient's clinical history. Thyroid stimulating hormone (TSH) receptor antibodies (TRAb) are typically negative, whereas anti-thyroglobulin (anti-TG) and antithyroxin peroxidase (anti-TPO) antibodies may be detectable. In selected cases, for example in patients with severe or prolonged hyperthyroidism, further examinations by ultrasound and scintigraphy might be necessary to confirm thyroid destruction and exclude an overactive thyroid as seen in Grave's disease or autonomous thyroid nodules. Ultrasound features of destructive thyroiditis are diffuse hypoechogenicities and a lack of hypervascularization. In scintigraphy a reduced uptake of technetium or iodine is characteristic for destructive thyroid disease [8]. Treatment of symptomatic thyrotoxicosis consists of beta-blockers, whereas levothyroxine is used for the substitution of clinically relevant hypothyroidism.

Due to the lack of prognostic benefits, high-dose glucocorticoid therapy is not recommended for routine treatment of ICI-induced thyroiditis and should be reserved only for exceptional cases of severe thyrotoxicosis or severe thyroid-related eye disease $[9,10]$. It is important to emphasize that the development of thyroid dysfunction is not a contraindication against the continuation of ICI therapy. On the contrary, an improved treatment response and survival rate has been reported for patients with thyroid irAEs although these data could be influenced by an immortal time bias [11]. Thyroiditis and other irAE might still be surrogates for the anti-tumoral immune-response. On the other hand, increasing evidence links thyroid hormone action with cancer proliferation by promoting growth, differentiation and metabolism [12], while pre-existing hypothyroidism in patients with cancer is associated with favorable survival $[13,14]$. At the moment it is unclear to what extent subclinical or overt hypothyroidism should be treated in cancer patients with ICI-related thyroid disease, since a hypothyroid state might actually be beneficial. Evidence from prospective, controlled studies are still missing and are urgently needed to answer this question. In our opinion, the indication for the substitution of subclinical hypothyroidism (TSH 4-10) following destructive thyroiditis in cancer patients should be based on individual clinical symptom relief rather than on arbitrarily defined cut-offs of hormonal values. There is no evidence that overt hypothyroidism should not be treated; however, this could change in the future.

\section{Hypophysitis}

Whereas hypophysitis is a rare disease in the general population, the prevalence reaches up to $17 \%$ in patients receiving ICI therapy, with a predominance in elderly patients and men [15]. The pathophysiological mechanisms still not completely understood. However, a role of ICI-induced antibodies against the anterior pituitary has been suggested $[2,16]$. Clinical pre- sentation is variable and ranges from asymptomatic laboratory abnormalities-mild hyponatremia is often observed, to severe fatigue and headache, whereas visual problems or polyuria/polydipsia are only rarely observed. Adrenocorticotropic hormone (ACTH) deficiency is the most frequent endocrine abnormality and might result in profound hypocortisolism [17]. As hypocortisolism might only become symptomatic, but then rapidly life-threatening, under acute stress circumstances, systematic biochemical screening is recommended by the European Endocrine Society (guidelines presented at the ECE 2021 and currently in preparation for publication) by testing morning cortisol and ACTH levels together with TSH, fT4 and electrolytes. In addition, luteinizing hormone (LH), follicle-stimulating hormone (FSH) and sex hormones (estrogen/testosterone) could help strengthen the diagnosis [9, 10]. Furthermore, pituitary MRI is recommended to rule out brain metastasis, whereas classical radiological signs of hypophysitis are only observed in about half of patients [16]. Treatment of hypophysitis most importantly consists in the substitution of corticotroph deficiency. Treatment of thyrotropin deficiency is less urgent and should be initiated only after glucocorticoid substitution therapy has been implemented to avoid adrenal crisis. Gonadal function can be replaced in selected patients depending on age and prognosis, whereas growth hormone treatment is generally contraindicated. Highdose glucocorticoid therapy is only reserved for patients suffering from mass effects (compression of the optic chiasma) or severe headache, but should not be routinely used $[9,10,18]$. Of note, normally pituitary hormone deficiency does not recover; therefore, it is essential to educate patients and their families in dose adaptations of glucocorticoid replacement in case of stressful situations or acute medical events (sick day rules). Patients should also be equipped with an emergency card.

However, regular testing for cortisol levels in asymptomatic patients with ICI therapy will inevitably give inconclusive results, as corticotroph deficiency can only be confirmed or ruled out by very low or high baseline cortisol concentrations, respectively. In addition, concomitant treatment with glucocorticoids for other indications like pain or nausea is frequent in cancer patients and might bias the hormone measurements. Dynamic testing by an ACTH stimulation test is recommended in this case $[9,10]$, but might not always be reliable, since in the very early phase of corticotropic deficiency the test might give a falsepositive response.

In selected cases a CRH stimulation test or an insulin hypoglycemia test might therefore be preferable, although more time and cost intensive (i.e., CRH test), or less-well tolerated and stressful for the patient (i.e., insulin hypoglycemia test).

A reasonable approach might be thorough education on potential clinical signs, laboratory findings 
(hyponatremia) and symptoms for patients, their relatives and their treating physicians for early detection of adrenal insufficiency and to perform diagnostic and treatment by glucocorticoid substitution as soon as clinically indicated. This approach is also in line with clinical practice guidelines from the European Society for Medical Oncology [19]. In case of uncertainty or under acute circumstances, it is strongly recommended to treat any patient who received ICI treatment for a potential adrenal crisis by stress doses of hydrocortisone until the diagnosis has been confirmed or ruled out [20].

Similar to thyroid irAEs, the development of hypophysitis during ICI therapy might be an indicator for treatment response, as studies link it with improved prognosis [21]. ICI therapy can be regularly continued, despite in the very rare case of severe mass effects in which treatment might be delayed $[9,10]$.

\section{Rare endocrine adverse events}

The development of type 1 diabetes mellitus as an irAE is rare, but severe and possibly life-threatening. Most cases have been reported following PD-1 directed treatment [1]. It is characterized by an acute onset, which results from a rapid insulin deficiency with a high risk of diabetic ketoacidosis [18]. Due to the fulminant onset, Hbalc is usually not helpful in establishing the diagnosis. The pathogenesis of ICIinduced diabetes mellitus is still unclear; however, destruction of insulin-secreting pancreatic beta cells is assumed. Classical auto-antibodies (including GADA, IAA, IA2, and anti-ZnT8) are positive in about half of patients and should be screened for. In addition, low concentrations of insulin and C-peptide confirm beta cell failure in the presence of hyperglycemia [1, 18].

Treatment of patients with ICI-induced diabetes mellitus is comparable to patients with type 1 diabetes mellitus and should initially consist of intensive insulin therapy and hydration. The guidelines for treating diabetic ketoacidosis apply. In the long-run, treatment with long- and short-acting insulins is usually needed. Targets for glycemic control should be adapted to each patient's comorbidities and overall prognosis $[9,10]$.

Another rare irAE is primary adrenal insufficiency $[3,9,22]$. Clinical signs appear to be similar to hypocortisolism in hypophysitis, although hypovolemia and electrolyte disorders (hyponatremia plus hyperkalemia) might be more pronounced due to the concomitant mineralocorticoid insufficiency. Diagnosis is based on paired serum cortisol and ACTH levels, as well as adrenal imaging to exclude bilateral metastases. Treatment should consist of glucocorticoid and mineralocorticoid replacement therapy in a standard dose, identical to non-iatrogenic primary adrenal insufficiency. Moreover, patients and their families must be educated to adapt their glucocorticoid replacement dose in case of stressful situations or acute medical events (sick day rules) [9, 10]. An emergency card must be issued for all patients.

Finally, single cases of primary hypoparathyroidism as an irAE following ICI therapy have been reported $[23,24]$. The pathogenesis might be explained by the development of activating antibodies of the calcium sensing receptor [25]. This results in suppression of parathyroid hormone secretion, which is followed by hypocalcemia. The diagnosis is confirmed by a low or inadequately normal PTH in the presence of albumincorrected hypocalcemia. In addition, testing for phosphate, magnesium, and 25-OH vitamin D should be performed. Treatment consists of calcium and bio-active vitamin D, similar to conventional hypoparathyroidism [26].

Funding Open access funding provided by Medical University of Vienna.

Conflict of interest P. Wolf and T. Scherer declare that they have no competing interests.

Open Access This article is licensed under a Creative Commons Attribution 4.0 International License, which permits use, sharing, adaptation, distribution and reproduction in any medium or format, as long as you give appropriate credit to the original author(s) and the source, provide a link to the Creative Commons licence, and indicate if changes were made. The images or other third party material in this article are included in the article's Creative Commons licence, unless indicated otherwise in a credit line to the material. If material is not included in the article's Creative Commons licence and your intended use is not permitted by statutory regulation or exceeds the permitted use, you will need to obtain permission directly from the copyright holder. To view a copy of this licence, visit http://creativecommons.org/licenses/by/4.0/.

\section{References}

1. ChangLS, Barroso-Sousa R, TolaneySM,HodiFS, KaiserUB, Min L. Endocrine toxicity of cancer immunotherapy targeting immune checkpoints. Endocr Rev. 2019;40(1):17-65.

2. Martins F, Sofiya L, Sykiotis GP, Lamine F, Maillard M, Fraga M, et al. Adverse effects of immune-checkpoint inhibitors: epidemiology, management and surveillance. NatRev Clin Oncol. 2019;16(9):563-80.

3. Corsello SM, Barnabei A, Marchetti P, De Vecchis L, Salvatori R, Torino F. Endocrine side effects induced by immune checkpoint inhibitors. J Clin Endocrinol Metab. 2013;98(4):1361-75.

4. Khoja L, Day D, Wei-Wu Chen T, Siu LL, Hansen AR. Tumour-and class-specific patterns ofimmune-related adverse events of immune checkpoint inhibitors: a systematic review. Ann Oncol. 2017;28(10):2377-85.

5. Abdel-Rahman O, ElHalawani H, Fouad M. Risk of endocrine complications in cancer patients treated with immune check point inhibitors: a meta-analysis. Future Oncol. 2016;12(3):413-25.

6. Bai X, Chen X, Wu X, Huang Y, Zhuang Y, Lin X. Immune checkpoint inhibitor-associated thyroid dysfunction: a disproportionalityanalysisusing theWHOAdverse DrugReaction Database, VigiBase. Eur JEndocrinol. 2020;182(1):1-9.

7. Delivanis DA, Gustafson MP, Bornschlegl S, Merten MM, Kottschade L, Withers S, et al. Pembrolizumab-induced thyroiditis: comprehensive clinical review and insights into 
underlying involved mechanisms. JClin Endocrinol Metab. 2017;102(8):2770-80.

8. Muir CA, Clifton-Bligh RJ, Long GV, Scolyer RA, Lo SN, Carlino MS, et al. Thyroid immune-related adverse events following immune checkpoint inhibitor treatment. J Clin Endocrinol Metab. 2021;106(9):e3704-e13.

9. Stelmachowska-Banaś M, Czajka-Oraniec I. Management of endocrine immune-related adverse events of immune checkpointinhibitors: an updated review. Endocr Connect. 2020;9:207-28.

10. Castinetti F, Albarel F, Archambeaud F, Bertherat J, Bouillet B, Buffier P, et al. French endocrine society guidance on endocrine side effects of immunotherapy endocrinerelated. Endocr Relat Cancer. 2019;26(2):G1-G18.

11. Kotwal A, Kottschade L, Ryder M. PD-L1 inhibitor-induced thyroiditis is associated with better overall survival in cancer patients. Thyroid. 2020;30(2):177-84.

12. Moeller LC, Führer D. Thyroid hormone, thyroid hormone receptors, and cancer: a clinical perspective. Endocr Relat Cancer. 2013;20(2):R19-R29.

13. Berghoff AS, Wippel C, Starzer AM, Ballarini N, Wolpert F, Bergen E, et al. Hypothyroidism correlates with favourable survival prognosis in patients with brain metastatic cancer. Eur JCancer. 2020;135:150-8.

14. Lechner MG, Vyas CM, Hamnvik O-PR, Alexander EK, Larsen PR, Choueiri TK, et al. Hypothyroidism during tyrosine kinase inhibitor therapy is associated with longer survival in patients with advanced nonthyroidal cancers. Thyroid. 2018;28(4):445-53.

15. Faje AT, Sullivan R, Lawrence D, Tritos NA, Fadden R, Klibanski A, et al. Ipilimumab-induced hypophysitis: a detailed longitudinal analysis in a large cohort of patients with metastatic melanoma. J Clin Endocrinol Metab. 2014;99(11):4078-85.

16. Albarel F, Castinetti F, Brue T. Immune check point inhibitors-induced hypophysitis. Eur J Endocrinol. 2019;181:107-18.

17. DiDalmazi G, Ippolito S, Lupi I, Caturegli P. Hypophysitisinduced by immune checkpoint inhibitors: a 10-year assessment. Expert RevEndocrinol Metab. 2019;14(6):381-98.

18. Paschou SA, Stefanaki K, Psaltopoulou T, Liontos M, Koutsoukos K, Zagouri F, et al. How we treat endocrine complications of immune checkpoint inhibitors. ESMO Open. 2021;6(1):100011.

19. Haanen JBAG, Carbonnel F, Robert C, Kerr KM, Peters S, Larkin J, et al. Management of toxicities from immunotherapy: ESMO Clinical Practice Guidelines for diagnosis, treatment and follow-up. Ann Oncol. 2017;28(suppl_4):iv119-iv42.

20. Higham CE, Olsson-Brown A, Carroll P, Cooksley T, Larkin J, Lorigan $\mathrm{P}$, et al. Acute management of the endocrine complications of checkpoint inhibitor therapy. Endocr Connect. 2018;7(7):G1-G7.

21. Matsuoka H, Hayashi T, Takigami K, Imaizumi K, Shiroki R, Ohmiya N, et al. Correlation between immune-related adverse events and prognosis in patients with various cancers treated with anti PD- 1 antibody. BMC Cancer. 2020;20(1):656.

22. Tan MH, Iyengar R, Mizokami-Stout K, Yentz S, MacEachern MP, Shen LY, et al. Spectrum of immune checkpoint inhibitors-induced endocrinopathies in cancer patients: a scoping review of case reports. Clin Diabetes Endocrinol. 2019;5(1):1.

23. Trinh B, Sanchez GO, Herzig P, Läubli H. Inflammation-induced hypoparathyroidism triggered by combination immune checkpoint blockade for melanoma. J Immunother Cancer. 2019;7(1):52.

24. Win MA, Thein KZ, Qdaisat A, Yeung S-CJ. Acute symptomatic hypocalcemia from immune checkpoint therapy-induced hypoparathyroidism. Am J Emerg Med. 2017;35(7):1039.e5-1039.e7.

25. Piranavan P, Li Y, Brown E, Kemp EH, Trivedi N. Immune checkpoint inhibitor-induced hypoparathyroidism associated with calcium-sensing receptor-activating autoantibodies. JClin Endocrinol Metab. 2018;104(2):550-6.

26. Bollerslev J, Rejnmark L, Marcocci C, Shoback DM, SitgesSerraA, van Biesen W, et al. European Society of Endocrinology Clinical Guideline: treatment of chronic hypoparathyroidismin adults. Eur JEndocrinol. 2015;173(2):G1-G20.

Publisher's Note Springer Nature remains neutral with regard to jurisdictional claims in published maps and institutional affiliations.

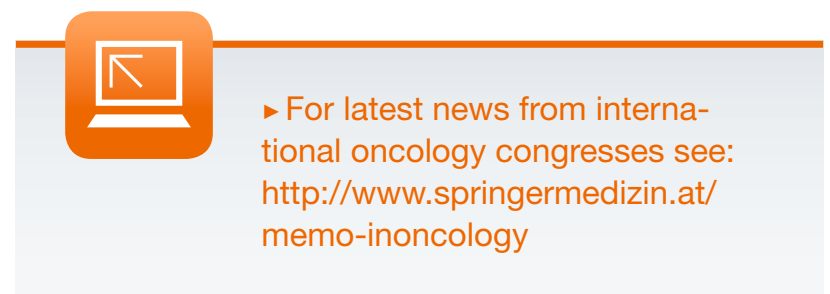

